\section{Prevalência e fatores associados ao sedentarismo no lazer em adultos}

\author{
Prevalence and variables associated \\ with leisure-time sedentary lifestyle in adults
}

\author{
1 Departamento de Educação \\ Física, Universidade Federal \\ da Bahia, Salvador, Brasil. \\ 2 Instituto de Saúde Coletiva, \\ Universidade Federal da \\ Bahia, Salvador, Brasil. \\ Correspondência \\ F. J. G. Pitanga \\ Departamento de Educação \\ Física, Universidade \\ Federal da Bahia. \\ Av. Luiz Tarquínio Pontes \\ 600, Lauro de Freitas, BA \\ 42700-000, Brasil. \\ pitanga@lognet.com.br
}

\section{Abstract}

This study focused on the prevalence and determinants of leisure-time sedentary lifestyle in the city of Salvador, Bahia, Brazil. A cross-sectional design was used in a sample of 2,292 adults $\geq 20$ years of age, of whom 1,271 (55.0\%) were females. Leisure-time sedentary lifestyle was defined by individuals who, in a live interview, stated that they performed no physical activity during their leisure time in a normal week. Initially, total prevalence of leisure-time sedentary lifestyle in the study population was calculated by variables associated and stratified by sex. Then, the prevalence ratio between leisure-time sedentary lifestyle, age, schooling, and marital status stratified by sex was calculated. A 95\% confidence interval was used. Prevalence of leisuretime sedentary lifestyle was $72.5 \%$ and was more frequent in women 40-50 years of age and men over 60, individuals with limited schooling, and married, separated, and widowed individuals. The findings are relevant for public health, since they can be used both to identify high levels of leisure-time sedentary lifestyle in the Brazilian population as well as the determinants, thus allowing new intervention strategies to be implemented.

Leisure Activities; Motor Activity; Life Style; Adults
Francisco José Gondim Pitanga 1

Ines Lessa ${ }^{2}$

\section{Introdução}

Com o processo da industrialização, existe um crescente número de pessoas que se tornam sedentárias com poucas oportunidades de praticar atividades físicas. Diversos autores têm demonstrado associação entre sedentarismo e agravos cardiovasculares 1, câncer 2,3, diabetes 4 e saúde mental 5 . Outros estudos demonstram que o sedentarismo no lazer está associado à hipertensão arterial e diabetes $6,7,8$, além de ser mais prevalente em mulheres, idosos e pessoas de baixa escolaridade 9,10 .

Sedentarismo no lazer pode ser identificado como a não participação em atividades físicas nos momentos de lazer, considerando atividade física como qualquer movimento corporal produzido pela musculatura esquelética que resulte em gasto energético 11 , tendo componentes e determinantes de ordem bio-psicosocial, cultural e comportamental, podendo ser exemplificada por jogos, lutas, danças, esportes, exercícios físicos e deslocamentos.

Para identificar o sedentarismo, o instrumento que vem sendo utilizado de forma mais freqüente é a versão curta do Questionário Internacional de Atividade Física (QIAF), que contempla as diversas facetas desse problema de saúde pública: atividades domésticas, atividades no trabalho, atividades no lazer e deslocamentos 12 . Assim, o sedentarismo no lazer é um recorte do sedentarismo quando analisado globalmente. 
O conhecimento de dados sobre o sedentarismo no lazer e seus determinantes, ou fatores associados, traz importantes contribuições para a saúde pública, porque pode servir de base para o gerenciamento de atividades de incentivo à prática de atividades físicas em subgrupos populacionais mais afetados por esse tipo de comportamento.

Este trabalho teve como objetivo verificar a prevalência e determinantes do sedentarismo no lazer em adultos na cidade de Salvador, Bahia, Brasil.

\section{Metodologia}

Estudo transversal realizado em Salvador, em 2000, com participantes do projeto Monitoramento das Doenças Cardiovasculares e do Diabetes no Brasil (MONIT), desenvolvido pela equipe de doenças crônicas não transmissíveis do Instituto de Saúde Coletiva (ISC) da Universidade Federal da Bahia (UFBA) e financiado pelo Ministério da Saúde do Brasil, que teve como objetivo determinar a prevalência dos fatores de risco cardiovascular na cidade de Salvador 13 .

Para coleta dos dados do projeto MONIT, foi utilizado o censo domiciliar realizado por investigadores do projeto Bahia Azul, em desenvolvimento por pesquisadores do ISC/UFBA e direcionado a outros objetivos 14 .

\section{Amostra}

A amostra foi probabilística por conglomerados, estimada com base em prevalência de hipertensão arterial de $25,0 \%$, nível de confiança de $95,0 \%$ e erro de delineamento de $2,0 \%$, sendo feita em três etapas: (1) os setores censitários de 8 das 10 bacias hidrográficas da cidade, com características sócio-demográficas semelhantes, foram agrupados em "Áreas de Pesquisa” (108 áreas), e essas, classificadas por nível sócio-econômico alto, misto e baixo. Ao todo, as áreas continham 16.592 domicílios, com aproximadamente 83 mil habitantes $\geq 20$ anos, sorteando-se probabilisticamente 37 delas, proporcionalmente ao número de setores de cada nível sócio-econômico; (2) nesse estágio, foram sorteados 1.540 domicílios, por amostra sistemática (intervalo $=10$ ), com resposta favorável à participação de 1.258 famílias $(81,7 \%)$ residentes em 63 setores censitários. As famílias que se recusaram a participar do estudo concentravam-se predominantemente no ní- vel sócio-econômico alto; (3) no terceiro estágio, foram sorteados os participantes, no máximo, dois por domicílio, um de cada sexo. Foram programadas 2.476 entrevistas, havendo $2,9 \%$ de recusas (72) e uma perda irrecuperável de 4,3\% (107) questionários completos. Na seqüência, foram excluídos do estudo quatro homens e uma mulher que não responderam, por completo, o questionário utilizado para a coleta de dados. Dessa forma, a amostra foi constituída por 2.292 adultos com idade entre 20 a 94 anos de idade, sendo 1.021 do sexo masculino e 1.271 do sexo feminino.

\section{Variáveis de estudo}

As variáveis de estudo foram: sedentarismo no lazer (variável dependente), idade, sexo, nível de escolaridade e estado civil (variáveis independentes).

\section{Coleta dos dados}

Dez entrevistadores de campo e duas supervisoras foram devidamente treinados para todas as etapas do trabalho. Para testes e correções dos instrumentos e técnicas, inclusive da dinâmica do trabalho de campo, cinqüenta residências (cem participantes) foram visitadas, e as entrevistas, realizadas seguindo toda a metodologia proposta. O grupo teste não se inclui na amostra. Todos os participantes da pesquisa foram entrevistados em domicílio para coleta dos dados demográficos e sobre sedentarismo no lazer. Com relação às atividades físicas no lazer, a pergunta foi feita da seguinte forma: Como você classificaria sua atividade física de lazer? (1) leve - caminhar, pedalar ou dançar mais ou igual que três horas por semana; (2) moderada - correr, fazer ginástica ou praticar esportes mais ou igual que três horas por semana; (3) intensa - treinamento para competição e (4) não tem - o lazer não inclui atividade física. Foram considerados como sedentários no lazer aqueles que informaram não participar de atividades físicas nos momentos de lazer, considerando a semana típica habitual.

\section{Procedimentos de análise}

Inicialmente, calculou-se a prevalência do sedentarismo no lazer na população estudada e nos diversos estratos de cada uma das variáveis analisadas (sexo, idade, escolaridade e estado civil). Na seqüência, calculou-se a prevalência 
do sedentarismo no lazer nos diversos estratos das variáveis analisadas controlando por sexo. Utilizou-se o teste do qui-quadrado para identificação das diferenças entre os estratos das variáveis estudadas. Posteriormente, calculouse a razão de prevalência entre sedentarismo no lazer, idade, nível de escolaridade e estado civil controlando por sexo. Nessa etapa da análise, a variável sexo foi trabalhada de forma dicotômica: sexo $=0$ se homem e sexo $=1$ se mulher. As outras variáveis foram divididas em estratos: idade $=0$ se $<40$ anos, idade $=1$ se entre 40 e 59 anos e idade $=2$ se $\geq 60$ anos; escolaridade $=0$ se universitário ou segundo grau completo, escolaridade $=1$ se primeiro grau completo ou segundo grau incompleto e escolaridade $=2$ se analfabeto ou até a 4 a série do primeiro grau; sedentário no lazer $=0$ se participa de atividades físicas nos momentos de lazer e sedentário no lazer $=1$ se não participa de atividades físicas nos momentos de lazer; estado civil $=0$ se solteiro, estado civil $=1$ se casado $\mathrm{e}$ estado civil = 2 se separado, viúvo e outros. Ainda, nesta análise, foram criadas variáveis dummies para comparação entre o grupo de referência $=0$ e os outros estratos de cada variável estudada. As diferenças foram observadas através do teste de Mantel-Hanzel. Utilizou-se o intervalo de confiança a $95 \%$. Todas as análises foram realizadas levando-se em consideração o efeito de delineamento amostral (conglomerados). A ponderação foi feita considerando a unidade amostral domicílios. Os dados foram analisados através do programa estatístico Stata, versão 7.0.
O projeto foi apresentado ao Comitê de Ética do Conselho Regional de Medicina do Estado da Bahia, sendo aprovado na íntegra. Todos os participantes do estudo ou seus responsáveis assinaram termo de consentimento concordando em participar da pesquisa.

\section{Resultados}

As características da amostra são apresentadas na Tabela 1. Observa-se que existem diferenças entre o sexo masculino e o feminino na média da idade e nas proporções dos diferentes estratos da variável estado civil. Com relação ao nível de escolaridade, não existem diferenças entre sexos nos diversos estratos de escolarização.

As prevalências do sedentarismo no lazer em cada um dos estratos das diversas variáveis analisadas, bem como na população total do estudo encontram-se na Tabela 2. Para sexo e escolaridade, o maior percentual de sedentarismo no lazer foi observado entre as mulheres e entre o grupo dos analfabetos até a 4 a série do primeiro grau. Quanto à idade e ao estado civil, o sedentarismo no lazer predominou na faixa etária de 40-59 anos e entre os casados, separados e viúvos, com diferenças estatisticamente significativas entre os diversos estratos dessas variáveis.

As prevalências do sedentarismo no lazer em cada um dos estratos das diversas variáveis analisadas estratificadas por sexo estão apresentadas na Tabela 3. Com relação à variável idade, entre os homens, observa-se associação

Média, desvio padrão e valores percentuais das variáveis analisadas no estudo. Salvador, Bahia, Brasil, 2000.

\begin{tabular}{|c|c|c|c|}
\hline & Homens $(n=1.021)$ & Mulheres $(n=1.271)$ & p ou $\chi^{2}$ \\
\hline \multirow[t]{2}{*}{ Idade (anos) } & $39,9 \pm 14,4$ & $41,7 \pm 14,9$ & \\
\hline & $(20-90)$ & $(20-94)$ & 0,002 \\
\hline \multicolumn{4}{|l|}{ Estado civil (\%) } \\
\hline Solteiros & 28,0 & 21,9 & \\
\hline Casados & 67,8 & 61,4 & \\
\hline Separados/viúvos/outros & 4,2 & 16,7 & 0,05 \\
\hline \multicolumn{4}{|l|}{ Escolaridade (\%) } \\
\hline 2o grau completo/universitário & 5,3 & 4,8 & \\
\hline 1ㅇ grau completo/2o grau incompleto & 53,5 & 51,0 & \\
\hline Analfabeto/até 4a série do 1o grau & 41,2 & 44,2 & 0,91 \\
\hline
\end{tabular}

Valores contínuos foram comparados através do teste " $t$ " de student para amostras independentes, e valores percentuais, através do teste qui-quadrado; $\chi^{2}=$ teste do qui-quadrado. 
Prevalência do sedentarismo no lazer por fatores associados. Salvador, Bahia, Brasil, 2004.

\begin{tabular}{|c|c|c|c|c|c|c|c|}
\hline \multirow[t]{3}{*}{ Variáveis } & \multicolumn{6}{|c|}{ Sedentarismo no lazer (\%) } & \multirow{3}{*}{$\chi^{2}$} \\
\hline & \multicolumn{3}{|c|}{ Sedentários } & \multicolumn{3}{|c|}{ Ativos } & \\
\hline & $\mathrm{n}$ & $\mathrm{p}$ & IC95\% & $\mathrm{n}$ & p & IC95\% & \\
\hline \multicolumn{8}{|l|}{ Sexo } \\
\hline Homens & 617 & 60,4 & $(60,3-60,5)$ & 404 & 39,6 & $(39,5-39,7)$ & \\
\hline Mulheres & 1.051 & 82,7 & $(82,6-82,8)$ & 220 & 17,3 & $(17,2-17,4)$ & 0,00 \\
\hline \multicolumn{8}{|l|}{ Idade (anos) } \\
\hline $20-39$ & 778 & 66,1 & $(66,0-66,2)$ & 399 & 33,9 & $(33,8-40,0)$ & \\
\hline $40-59$ & 656 & 79,4 & $(79,3-79,5)$ & 170 & 20,6 & $(20,4-20,8)$ & \\
\hline$\geq 60$ & 225 & 77,7 & $(77,6-77,8)$ & 64 & 22,3 & $(22,0-22,6)$ & 0,05 \\
\hline \multicolumn{8}{|l|}{ Escolaridade } \\
\hline 2o grau completo e universitário & 53 & 46,3 & $(46,0-46,6)$ & 62 & 53,7 & $(53,4-54,0)$ & \\
\hline 1ㅇ grau completo e 2 o grau incompleto & 783 & 66,1 & $(66,0-66,2)$ & 402 & 33,9 & $(33,8-34,0)$ & \\
\hline Analfabeto/até 4a série do 1o grau & 808 & 82,7 & $(82,6-82,8)$ & 169 & 17,3 & $(17,1-17,5)$ & 0,00 \\
\hline \multicolumn{8}{|l|}{ Estado civil } \\
\hline Solteiros & 350 & 62,2 & $(62,1-62,3)$ & 212 & 37,8 & $(37,6-38,0)$ & \\
\hline Casados & 1.106 & 75,2 & $(75,1-75,3)$ & 365 & 24,8 & $(24,7-24,9)$ & \\
\hline Separados, viúvos e outros & 202 & 78,9 & $(78,7-79,1)$ & 54 & 21,1 & $(20,8-21,4)$ & 0,02 \\
\hline Total & 1.662 & 72,5 & $(72,4-72,6)$ & 630 & 27,5 & $(27,4-27,6)$ & \\
\hline
\end{tabular}

Tabela 3

Prevalência do sedentarismo no lazer por fatores associados estratificados por sexo. Salvador, Bahia, Brasil, 2004.

\begin{tabular}{|c|c|c|c|c|c|c|c|}
\hline \multirow[t]{3}{*}{ Variáveis } & \multicolumn{6}{|c|}{ Sedentarismo no lazer (\%) } & \multirow{3}{*}{$\chi^{2}$} \\
\hline & \multicolumn{3}{|c|}{ Masculino } & \multicolumn{3}{|c|}{ Feminino } & \\
\hline & $\mathrm{n}$ & $\mathrm{p}$ & IC95\% & $\mathrm{n}$ & $\mathrm{p}$ & IC95\% & \\
\hline \multicolumn{8}{|l|}{ Idade (anos) } \\
\hline $20-39$ & 270 & 49,2 & $(49,0-49,4)$ & 512 & 81,6 & $(81,5-81,7)$ & \\
\hline $40-59$ & 264 & 72,4 & $(72,2-72,6)$ & 395 & 85,2 & $(85,1-85,3)$ & \\
\hline$\geq 60$ & 83 & 74,8 & $(74,6-75,0)$ & 144 & 79,5 & $(79,3-79,7)$ & 0,16 \\
\hline \multicolumn{8}{|l|}{ Escolaridade } \\
\hline 2o grau completo e universitário & 22 & 40,5 & $(39,9-41,1)$ & 31 & 51,4 & $(50,9-51,9)$ & \\
\hline 1ㅇ grau completo e 2 o grau incompleto & 272 & 50,3 & $(50,1-50,3)$ & 516 & 80,2 & $(80,1-80,3)$ & \\
\hline Analfabeto/até 4a série do 1o grau & 316 & 75,4 & $(75,2-75,6)$ & 494 & 88,5 & $(88,4-88,6)$ & 0,42 \\
\hline \multicolumn{8}{|l|}{ Estado civil } \\
\hline Solteiros & 129 & 45,5 & $(45,3-45,7)$ & 224 & 80,7 & $(80,6-80,8)$ & \\
\hline Casados & 460 & 66,6 & $(66,5-66,8)$ & 650 & 83,3 & $(83,2-83,5)$ & \\
\hline Separados, viúvos e outros & 25 & 58,3 & $(57,8-58,8)$ & 177 & 83,0 & $(82,8-83,2)$ & 0,32 \\
\hline Total & 617 & 60,4 & $(60,3-60,5)$ & 1051 & 82,7 & $(82,6-82,8)$ & \\
\hline
\end{tabular}


positiva com sedentarismo no lazer; entre as mulheres, observa-se aumento da prevalência do sedentarismo no lazer até o estrato de 40-59 anos, a partir do 60 anos, a prevalência do sedentarismo no lazer tende a diminuir. Para escolaridade, o maior percentual de sedentarismo no lazer foi observado entre os analfabetos, tanto para homens quanto para mulheres, sem diferenças estatisticamente significativas entre os diversos estratos dessas variáveis. Quanto ao estado civil, o sedentarismo no lazer predominou entre os casados nos homens e entre casados, separados e viúvos para mulheres, também sem diferenças estatisticamente significativas entre os diversos estratos dessa variável. Observa-se também que, no total, a prevalência do sedentarismo no lazer é maior entre mulheres do que entre homens.

As razões de prevalência do sedentarismo no lazer para idade, nível de escolaridade e estado civil estratificados por sexo estão na Tabela 4. Esses resultados demonstram que homens entre 40-59 anos de idade, pessoas de ambos os sexos com baixo nível de escolaridade, além de casados, separados e viúvos têm mais possibilidades de serem sedentários no lazer. Importante salientar que, entre mulheres ( $\geq 60$ anos), a idade parece ter um efeito protetor contra o sedentarismo no lazer.

\section{Discussão}

Analisando a população total do estudo, observam-se valores elevados de sedentarismo no lazer principalmente quando comparados com outros estudos demonstrando que, na população da cidade de Salvador, existe um grande número de pessoas que não praticam atividades físicas nos momentos de lazer.

Nos estudos internacionais sobre esse mesmo tema, com grandes amostras, as prevalências do sedentarismo no lazer variaram, sendo descritas desde $28,9 \%$ para mulheres e $24,2 \%$ para homens em países da União Européia, incluindo uma variação de $8,1 \%$ na Finlândia e $59,3 \%$ em Portugal 15. No Brasil, Gomes et al. 9 encontraram prevalência do sedentarismo no lazer de 58,9\% em homens e 77,8\% em mulheres, e Monteiro et al. 16, analisando 11.033 adultos de ambos os sexos, observaram sedentarismo no lazer em $87,0 \%$ dos participantes do estudo.

Recentemente, Costa et al. 10 analisaram 4.030 funcionários de uma universidade do Estado do Rio de Janeiro, encontrando prevalência de sedentarismo no lazer de $47,8 \%$ em homens e $59,2 \%$ em mulheres. Os resultados apresentados no nosso estudo demonstram tendên- cia similar, desde que, entre mulheres, a prevalência do sedentarismo no lazer é maior do que entre homens, apesar dos valores absolutos apresentarem tendência a serem maiores do que aqueles observados nas diversas populações estudadas.

Outros autores 17 identificaram a prevalência do sedentarismo analisado de forma global em 3.182 sujeitos na cidade de Pelotas, Brasil, encontrando o valor de $41,1 \%$. Importante salientar que, neste estudo, o sedentarismo foi analisado considerando atividades no lazer, atividades domésticas, atividades no trabalho e deslocamentos.

Com relação às características dos subgrupos populacionais mais afetados pelo sedentarismo no lazer, observou-se que mulheres de modo geral, homens entre 40-59 anos de idade, pessoas de ambos os sexos com baixo nível de escolaridade, além de casados, separados e viúvos têm menos possibilidades de envolvimento em atividades físicas durante as horas de lazer. Esses resultados são muito parecidos aos de Gomes et al. 9, que demonstraram, no Município do Rio do Janeiro, que os grupos de meia idade e idosos, as mulheres e os de baixa escolaridade apresentavam um maior risco de não realizar atividades físicas de lazer. Resultados similares foram também encontrados por Costa et al. 10 ao demonstrarem que o sedentarismo no lazer é mais prevalente em mulheres e associado ao baixo nível de escolaridade e idade.

Também Martinez-Gonzalez et al. 15, na Europa, observaram associação positiva entre sedentarismo no lazer e idade, baixo nível de escolaridade, estado civil casado e obesidade.

A associação observada entre sedentarismo no lazer e idade foi descrita por outros autores segundo os quais quanto maior a idade maiores possibilidades das pessoas se tornarem sedentárias no lazer 18. Um dado importante encontrado no nosso estudo é o efeito protetor da idade ( $>60$ anos) para sedentarismo no lazer em pessoas do sexo feminino. A explicação para esse fenômeno poderia ser o fato de que, nessa faixa etária, as mulheres já teriam criado seus filhos, muitas vezes, já estando aposentadas, tendo conseqüentemente mais oportunidades para participar de atividades físicas nos seus momentos de lazer.

Diversos trabalhos têm demonstrado associação entre sedentarismo no lazer e baixa escolaridade 19, identificando, inclusive, características importantes dos grupos de baixa escolaridade que predizem seus altos riscos para decréscimo na atividade física: baixa percepção de controle sobre a vida para todos os gru- 
Razão de prevalência entre sedentarismo no lazer, idade, grau de escolaridade e estado civil estratificados por sexo.

Salvador, Bahia, Brasil, 2004

\begin{tabular}{|c|c|c|c|c|}
\hline Variáveis & $\begin{array}{c}\text { Ambos os sexos } \\
\text { RP (IC95\%) }\end{array}$ & $\begin{array}{l}\text { Masculino } \\
\text { RP (IC95\%) }\end{array}$ & $\begin{array}{l}\text { Feminino } \\
\text { RP (IC95\%) }\end{array}$ & p valor \\
\hline \multicolumn{5}{|l|}{ Idade (anos) } \\
\hline $20-39$ & 1,00 & 1,00 & 1,00 & \\
\hline $40-59$ & $1,20(1,19-1,20)$ & $1,47(1,47-1,47)$ & $1,04(1,04-1,05)$ & 0,00 \\
\hline$\geq 60$ & $1,17(1,17-1,18)$ & $1,52(1,51-1,52)$ & $0,97(0,97-0,98)$ & 0,00 \\
\hline \multicolumn{5}{|l|}{ Escolaridade } \\
\hline 2o grau completo e universitário & 1,00 & 1,00 & 1,00 & \\
\hline 1ㅇ grau completo e 2 o grau incompleto & $1,43(1,42-1,44)$ & $1,24(1,23-1,26)$ & $1,56(1,55-1,57)$ & 0,26 \\
\hline Analfabeto/até 4a série do 1o grau & $1,79(1,78-1,80)$ & $1,86(1,84-1,88)$ & $1,72(1,71-1,73)$ & 0,90 \\
\hline \multicolumn{5}{|l|}{ Estado civil } \\
\hline Solteiros & 1,00 & 1,00 & 1,00 & \\
\hline Casados & $1,21(1,20-1,21)$ & $1,46(1,46-1,47)$ & $1,03(1,02-1,03)$ & 0,00 \\
\hline Separados, viúvos e outros & $1,27(1,26-1,27)$ & $1,28(1,27-1,29)$ & $1,03(1,02-1,03)$ & 0,50 \\
\hline
\end{tabular}

$\mathrm{RP}=$ razão de prevalência .

pos etários, além de problemas financeiros e baixa percepção geral da saúde para aqueles com mais de 45 anos de idade. Entretanto, deve-se ressaltar que, analisando o sedentarismo de forma global, a associação com escolaridade é inversa, ou seja, quanto menor o nível de escolaridade maior participação em atividades físicas 17. Essa tendência pode ser explicada em razão de que pessoas com menos escolaridade têm menos oportunidades de desenvolver atividades físicas nos momentos de lazer, enquanto que, ao ser analisado o sedentarismo de forma global, outras situações são levadas em consideração como: atividades no trabalho, atividades domésticas e deslocamentos. Dessa forma, pessoas como menor escolaridade passam a ter mais chances de serem identificadas como ativas fisicamente.

A relação entre o tipo de ocupação pouco qualificada e sedentarismo no lazer foi relatada por Lindström et al. 20, ao observarem que trabalhadores braçais, tanto homens quanto mulheres, têm mais probabilidades de serem sedentários no lazer do que trabalhadores não braçais. Observaram também que mulheres pensionistas e pessoas com pouca participação em atividades sociais são mais sedentárias nos momentos de lazer. Uma explicação para menor atividade física no lazer por pessoas com menor escolaridade seria sua inserção em atividades pesadas e não sedentárias no trabalho, conseqüentes à baixa qualificação, que é dependente da escolaridade. O inverso ocorre pa- ra pessoas com escolaridade mais elevada e atividade sedentária no trabalho.

Como o instrumento utilizado no estudo para análise do sedentarismo no lazer não foi construído especificamente para uma investigação sobre sedentarismo, mas para inquérito populacional com objetivo primário de identificar fatores de risco para doenças cardiovasculares e diabetes, essa pode ser uma limitação do estudo, embora os resultados sejam comparáveis aos da literatura. A análise para sedentarismo no lazer foi definida posteriormente à coleta de dados do estudo, não tendo sido validada nem testada a concordância teste-reteste do questionário com essa finalidade, embora tenha sido realizado o piloto para análise e reconstrução das questões de modo abrangente. Outros autores que estudaram sedentarismo no lazer utilizaram instrumentos muito parecidos com o nosso, como, por exemplo, através de questões como: "faz atividade física regular ou esportes?" 9, ou, "nas últimas duas semanas, você praticou alguma atividade física para melhorar sua saúde, condição física ou com objetivo estético ou de lazer?" 10 . A metodologia para análise do sedentarismo no lazer utilizada em trabalhos dessa natureza é rápida e conveniente para grandes estudos populacionais, porém, recomenda-se a inclusão de questões sobre duração e intensidade das atividades. A análise das variáveis determinantes do sedentarismo no lazer foi feita apenas de modo exploratório, não tendo sido observado se as associações de- 
tectadas persistiriam caso fossem ajustadas para possíveis variáveis de confundimento.

Os resultados apresentados são importantes para a saúde pública, na medida que podem ser utilizados para demonstrar os altos níveis de sedentarismo no lazer em adultos na cidade de Salvador, bem como para identificar fatores associados ou determinantes dessa situação. Desta forma, será possível implemen- tar programas de incentivo à prática de atividades físicas direcionados mais especificamente para subgrupos populacionais mais afetados pelo sedentarismo. Sugerem-se novos estudos que façam a análise tanto do sedentarismo no lazer quanto do sedentarismo de forma global, incluindo também as outras variáveis que compõem esse grave problema de saúde pública.

\section{Resumo}

O objetivo do estudo foi verificar a prevalência e determinantes do sedentarismo no lazer em adultos, na cidade de Salvador, Bahia, Brasil. O desenho foi transversal em amostra de 2.292 adultos $\geq 20$ anos de idade, sendo 1.271 (55,0\%) do sexo feminino. Sedentários no lazer foram aqueles que informaram não participar de atividades físicas nos momentos de lazer, em uma semana habitual. Inicialmente, calculou-se a prevalência do sedentarismo no lazer no total, por variáveis associadas e estratificadas por sexo na população estudada. Em seguida, calculou-se a razão de prevalência entre sedentarismo no lazer, idade, grau de escolaridade e estado civil, estratificados por sexo e intervalo de confiança a 95\%. A prevalência do sedentarismo no lazer foi de 72,5\%, sendo mais freqüente em mulheres entre 40-59 anos e homens maiores que 60 anos de idade, em pessoas com baixo nível de escolaridade e entre os casados, separados ou viúvos. Os resultados deste estudo são importantes para a saúde pública, porque podem ser utilizados para demonstrar os altos níveis de sedentarismo no lazer na nossa população, bem como para identificar seus determinantes, para que, desta forma, estratégias de intervenção possam ser implementadas.

Atividades de Lazer; Atividade Motora; Estilo de Vida; Adultos

\section{Colaboradores}

F. J. G. Pitanga realizou as etapas de análise dos dados e da revisão da literatura e redigiu o artigo. I. Lessa foi responsável pelo projeto que originou o banco de dados, coordenou a coleta dos dados e contribuiu na revisão do manuscrito.

\section{Agradecimentos}

Trabalho financiado pela Secretaria de Vigilância em Saúde, Ministério da Saúde do Brasil e Banco Mundial. 


\section{Referências}

1. Kohl HM. Physical activity and cardiovascular disease: evidence for a dose response. Med Sci Sports Exerc 2001; 33:472-83.

2. Friedenreich CM. Physical activity and cancer prevention: from observational to intervention research. Cancer Epidemiol Biomarkers Prev 2001; 10:287-301.

3. Thune I, Furberg AS. Physical activity and cancer risk: dose-response and cancer, all sites and sitespecific. Med Sci Sports Exerc 2001; 33:530-50.

4. Hu FB, Leitzmann MF, Stampfer MJ, Colditz GA, Willett WC, Rimm EB. Physical activity and television watching in relation to risk for type 2 diabetes mellitus in men. Arch Intern Med 2001; 161:1542-8.

5. Yaffe K, Barnes D, Nevitt M, Lui LY, Covinsky K. A prospective study of physical activity and cognitive decline in elderly women: women who walk. Arch Intern Med 2001; 161:1703-8.

6. Pitanga FJG, Lessa I. Associação entre sedentarismo no lazer e hipertensão arterial diastólica em adultos de ambos os sexos na cidade de SalvadorBA. In: Anais do 14o Congresso da Sociedade Brasileira de Hipertensão. Natal: Gráfica BG Cultural; 2003. p. 44.

7. Pitanga FJG, Lessa I. Associação entre sedentarismo no lazer e hipertensão arterial sistólica em adultos de ambos os sexos na cidade de SalvadorBA. In: Anais do 58o Congresso da Sociedade Brasileira de Cardiologia. Salvador: Gráfica Venture; 2003. p. 22.

8. Pitanga FJG, Lessa I. Associação entre sedentarismo no lazer e diabetes em adultos de ambos os sexos na cidade de Salvador-BA. In: Anais do XXVI Simpósio Internacional de Ciências do Esporte. São Paulo: Fontoura Editora; 2003. p. 174.

9. Gomes VB, Siqueira KS, Sichieri R. Atividade física em uma amostra probabilística da população do Município do Rio de Janeiro. Cad Saúde Pública 2001; 17:969-76.

10. Costa RS, Werneck GL, Lopes CS, Faerstein E. Associação entre fatores sócio-demográficos e prática de atividade física de lazer no estudo PróSaúde. Cad Saúde Pública 2003; 19:1095-105.

11. Caspersen CJ, Powell KE, Christenson GM. Physical activity, exercise and physical fitness. Public Health Rep 1985; 100:126-31.
12. Matsudo S, Timóteo A, Matsudo V, Andrade D, Andrade E, Oliveira LC, et al. Questionário Internacional de Atividade Física (IPAC): estudo de validade e reprodutibilidade no Brasil. Rev Bras Ativ Fís Saúde 2001; 6:5-18.

13. Centro Nacional de Epidemiologia/Fundação Nacional de Saúde. Projeto Monitoramento das Doenças Cardiovasculares e do Diabetes no Brasil (MONIT): relatório de pesquisa. Brasília: Instituto de Saúde Coletiva, Universidade Federal da Bahia; 2000.

14. Teixeira MG, Barreto ML, Costa MCN, Strina A, Martins Jr. D, Prado M. Sentinel areas: a monitoring strategy in public health. Cad Saúde Pública 2002; 18: 1189-95.

15. Martinez-Gonzalez MA, Varo JJ, Santos JI, De Irala J, Gibney M, Kearney J, et al. Prevalence of physical activity during leisure time in the Europe Union. Med Sci Sports Exerc 2001; 33:1142-6.

16. Monteiro CA, Conde WL, Matsudo SM, Matsudo VR, Bonseñor IM, Lotufo PA. A descriptive epidemiology of leisure-time physical activity in Brazil, 1996-1997. Rev Panam Salud Publica 2003; 14:246-54.

17. Hallal PC, Victora CG, Wells JC, Lima RC. Physical inactivity: prevalence and associated variables in Brazilian adults. Med Sci Sports Exerc 2003; 35: 1894-900.

18. Caspersen CJ, Pereira MA, Curran KM. Changes in physical activity patterns in the United States, by sex and cross-sectional age. Med Sci Sports Exerc 2000; 32:1601-9.

19. Droomers M, Schrijvers CT, Mackenbach JP. Educational level and decrease in leisure time physical activity: predictors from the longitudinal GLOBE study. J Epidemiol Community Health 2003; 55: 562-8.

20. Lindström M, Hanson BS, Östergren PO. Socioeconomic differences in leisure-time physical activity: the role of social participation and social capital in shaping health related behaviour. Soc Sci Med 2001; 52:441-51.

Recebido em 16/Jun/2004

Versão final reapresentada em 09/Dez/2004

Aprovado em 05/Jan/2005 